\title{
A sufficient conditions for global quadratic optimization
}

\author{
Mourad Naffouti ${ }^{1, *}$ and Abdeljelil Baccari ${ }^{2}$ \\ ${ }^{1}$ Faculty of Sciences of Tunis, University of Tunis El Manar \\ 2092 Tunis, Tunisia \\ E-mail: 〈mouradnaffouti@gmail.com〉 \\ 2 The Higher National Engineering School of Tunis, University of Tunis \\ 13 Ave Taha Hussein, Tunisia \\ E-mail: 〈abdbaccari@gmail.com〉
}

\begin{abstract}
This paper is devoted to global optimality conditions for quadratic optimization problems in a real space of dimension n. More precisely, we are concerned with nonconvex quadratic optimization problems with linear constraints. We present some sufficient conditions of global optimality for such problems subject to linear equality and inequality constraints. We prove that when the set of KarushKuhn-Tucker triplets of this problem is convex, then a local minimizer is global.
\end{abstract}

Keywords: convex sets, global optimality conditions, linear constraints, nonconvex quadratic optimization

Received: February 10, 2020; accepted: March 30, 2020; available online: July 07, 2020

DOI: 10.17535 /crorr.2020.0002

\section{Introduction}

Nonconvex quadratic optimization with linear constraints is a problem of minimization of nonconvex quadratic function subject to linear equality and inequality constraints. This problem is important in mathematical programming, and it has numerous applications in diverse fields of science and technology, see $[11,12]$ for more explanations. It is well known that any local minimum is global for a convex quadratic optimization problems, for more results on this topic, we refer the readers to Stephen Boyd and Lieven Vandenberghe [2] and Jorge Nocedal and Stephen Wright [8]. In general, a local minimizer may not be a global minimizer.

In this paper, we present some global optimality conditions for a nonconvex quadratic function subject to linear constraints. This problem is an NP-hard global optimization problem, see $[7,9,10]$. In $[5,8]$, the authors showed that, for some quadratic optimization problems with linear equality constraints, if the Jacobian matrix of its equality constraints has a fullrow rank and the Hessian matrix of the Lagrangian is positive definite on the null space of this Jacobian matrix, then there is a single global minimum. Without using this condition, we establish alternative theorems to certain global optimality problems, and we present global optimality conditions of a quadratic optimization problem with linear equality and inequality constraints. We prove that, if the set of Karush-Kuhn-Tucker (KKT) triplets is convex, then a local minimizer is global.

The outline of this paper is as follows. In the Section 2 we present notations and definitions, which include the firs-order and the second-order necessary optimality conditions for a quadratic optimization problem with linear constraints either equality or both equality and inequality. The main results are stated and proved in the Section 3. In the Section 4 we validate the obtained results by applying our approach to an example of quadratic optimization problems. Finally, conclusion is given in the Section 5.

*Corresponding author. 


\section{Preliminaries and definitions}

Consider the following quadratic optimization problem with linear equality constraints

$$
\begin{array}{ll}
\min _{x \in \mathbb{R}^{n}} & q(x)=\frac{1}{2} x^{T} Q x-c^{T} x, \\
\text { s. t. } & A x=b,
\end{array}
$$

where $x \in \mathbb{R}^{n}, Q \in \mathbb{R}^{n \times n}$, is a symmetric indefinite matrix; $x^{T}$ denotes the transposition of $x$; $A$ is an $\mathrm{m}$ by $\mathrm{n}$ real matrix with $m \leq n$, b is a constant vector of $\mathbb{R}^{m}$, c is a constant vector of $\mathbb{R}^{n}$ and

$$
\mathcal{F}=\left\{x \in \mathbb{R}^{n}: A x=b\right\},
$$

the feasible set of problem (P1)

Recall that $x^{*} \in \mathcal{F}$ is a local minimizer, for problem (P1), if and only if $x^{*}$ satisfies first-order and second-order necessary optimality conditions of Karush-Kuhn-Tucker, see for instance $[1,3]$. So $x^{*}$ is a local minimizer for the problem (P1), if and only if there exists a Lagrange multiplier $\lambda^{*} \in \mathbb{R}^{m}$, satisfies

$$
\begin{aligned}
\nabla_{x} L\left(x^{*}, \lambda^{*}\right) & =0, \\
\nabla_{\lambda} L\left(x^{*}, \lambda^{*}\right)= & 0, \\
d^{T} Q d \geq 0 & \forall d \in \operatorname{Ker}(A),
\end{aligned}
$$

where the Lagrangian function

$$
L(x, \lambda)=q(x)+\lambda^{T}(A x-b),
$$

is defined on $\mathbb{R}^{n} \times \mathbb{R}^{m} . \nabla_{x}, \nabla_{\lambda}$ denotes the gradient with respect to $x$ and $\lambda$, respectively and

$$
\operatorname{Ker}(A)=\left\{x \in \mathbb{R}^{n}: A x=0\right\} .
$$

This leads, in particular, to the following first order optimality conditions

$$
\begin{aligned}
Q x^{*}+A^{T} \lambda^{*} & =c, \\
A x^{*} & =b .
\end{aligned}
$$

Definition 1. A KKT pair for the problem (P1) is a pair $(x, \lambda) \in \mathbb{R}^{n} \times \mathbb{R}^{m}$ satisfying the equations (4) and (5) and $x$ is called a KKT point of problem (P1).

The set of the KKT pairs for the problem (P1) is denoted by $\mathcal{K}_{\mathcal{F}}$.

Consider next the following quadratic optimisation problem with linear equality and inequality constraints:

$$
\begin{array}{ll}
\min _{x \in \mathbb{R}^{n}} & q(x)=\frac{1}{2} x^{T} Q x-c^{T} x, \\
\text { s. t. } & A x=b, \\
& x_{i} \geq 0, \forall i \in\{1,2, \ldots, n\} .
\end{array}
$$

The feasible set of this problem is denoted by

$$
\Delta=\left\{x \in \mathbb{R}^{n} \mid A x=b, x_{i} \geq 0, i \in\{1,2, \ldots, n\}\right\} .
$$

The Lagrangian function is defined, on $\mathbb{R}^{n} \times \mathbb{R}^{m} \times \mathbb{R}^{n}$, by

$$
\mathcal{L}(x, \lambda, \mu)=\frac{1}{2} x^{T} Q x-c^{T} x+\lambda^{T}(A x-b)-\sum_{i=1}^{n} \mu_{i} x_{i} .
$$


It can be seen that the problem $(\mathrm{P} 2)$ is reduced to the problem $(\mathrm{P} 1)$, when there is no inequality constraints.

A feasible point $\bar{x} \in \Delta$ is said to be a KKT point (or a stationnary point for the Lagrangian function $\mathcal{L}$ ) of the problem (P2), if $\bar{x}$ satisfies necessary first-order optimality conditions of Karush-Kuhn-Tucker and there exists Lagrange multipliers $(\bar{\lambda}, \bar{\mu})$ such that

$$
\left\{\begin{array}{l}
Q \bar{x}-c+A^{T} \bar{\lambda}-\bar{\mu}=0, \\
A \bar{x}=b, \\
\bar{x}_{i} \geq 0, \bar{\mu}_{i} \geq 0, \bar{\mu}_{i} \bar{x}_{i}=0, \quad \forall i=1,2, \ldots, n .
\end{array}\right.
$$

Let $I(\bar{x})=\left\{i \in\{1,2, \ldots, n\}: \bar{x}_{i}=0\right\}$ be the index set of active constraints. We put

$$
M(\bar{x})=\left\{i \in\{1,2, \ldots, n\}: \bar{\mu}_{i}>0\right\} \subseteq I(\bar{x}),
$$

Recall that a triplet $(\bar{x}, \bar{\lambda}, \bar{\mu})$ satisfy the strict complementarity condition if $M(\bar{x})=I(\bar{x})$.

Let

$$
T_{\triangle}(\bar{x})=\left\{d \in \mathbb{R}^{n} \mid A d=0 ; d_{i} \geq 0, \forall i \in I(\bar{x})\right\},
$$

be the tangent cone to $\triangle$ at $\bar{x}$ and let $C(\bar{x})=T_{\triangle}(\bar{x}) \cap \nabla_{x} q(\bar{x})^{\perp}$ be the critical cone. One has

$$
C(\bar{x})=\left\{d \in \mathbb{R}^{n} \mid A d=0 ; d_{i} \geq 0, \forall i \in I(\bar{x}) \text { and } d_{i}=0 \text { for } \bar{\mu}_{i}>0\right\} .
$$

A KKT point $\bar{x} \in \Delta$ satisfies necessary second-order optimality conditions if the following conditions holds:

$$
d^{T} Q d \geq 0 \quad \forall d \in C(\bar{x})
$$

Definition 2. A KKT triplet for the problem (P2), is a triplet $(x, \lambda, \mu) \in \mathbb{R}^{n} \times \mathbb{R}^{m} \times \mathbb{R}^{n}$ satisfying the conditions (CN1) and $x$ is called a KKT point of problem (P29.

The set of the KKT triplets for (P2) is denoted by $\mathcal{K}_{\triangle}$.

In general, the set $\mathcal{K}_{\triangle}$ is not a convex set.

Definition 3. Let $J \subset\{1,2, \ldots, n\}$. The pseudo-face $\triangle_{J}$ is the subset of $\triangle$ defined by [6]

$$
\triangle_{J}=\left\{x \in \triangle \mid x_{j}=0, j \in J ; x_{j}>0, j \in\{1,2, \ldots, n\} \backslash J\right\} .
$$

By Contesse [3], it is well known that the conditions (CN1) and (CN2)are necessary and sufficient conditions for local optimality conditions for this problem.

For (P2), the most difficulty is that the local optimality does not imply the global optimality in general, but we will see that this implication holds in a special case. In the next section, we propose a global optimality criterion for this problem.

\section{Mains results}

In the following lemma, we present a first global optimality condition for the problem (P1)

Lemma 1. For the problem (P1), every local minimizer is a global minimizer.

Proof. Let $\bar{x}$ be a local minimizer for ( $\mathrm{P} 1, \bar{\lambda}$ is an associated Lagrange multiplier vector and $\mathrm{x}$ be a feasible point for this problem (satisfying $A x=b$ ). Then, by using the second-order Taylor expansion and the Hessian of the Lagrangian function, we have

$$
q(x)=L(x, \bar{\lambda})=L(\bar{x}, \bar{\lambda})+\nabla_{x} L(\bar{x}, \bar{\lambda})^{T}(x-\bar{x})+\frac{1}{2}(x-\bar{x})^{T} Q(x-\bar{x}) .
$$

As $L(\bar{x}, \bar{\lambda})=q(\bar{x}),\left(\nabla_{x} L(\bar{x}, \bar{\lambda})=0\right.$ and $(x-\bar{x}) \in \operatorname{Ker}(A)$, so from equation (3), we have that

$$
q(x) \geq q(\bar{x}) .
$$


The set $\mathcal{K}_{\mathcal{F}}$ is a convex set and has two properties listed in the following lemma.

Lemma 2. We have

1. $(y-x)^{T} Q(y-x)=0 \quad \forall\left\{\left(x, \lambda_{x}\right),\left(y, \lambda_{y}\right)\right\} \subset \mathcal{K}_{\mathcal{F}}$,

2. The objective function $q$ is constant over $\mathcal{K}_{\mathcal{F}}$.

\section{Proof.}

1. By applying $\left(x, \lambda_{x}\right)$ and $\left(y, \lambda_{y}\right)$ into (4), we have

$$
\begin{aligned}
& Q x-c+A^{T} \lambda_{x}=0, \\
& Q y-c+A^{T} \lambda_{y}=0 .
\end{aligned}
$$

Furthermore, by subtracting these equations, we obtain an expression of the form

$$
Q(y-x)+A^{T}\left(\lambda_{y}-\lambda_{x}\right)=0 .
$$

Then we have

$$
(y-x)^{T} Q(y-x)+(y-x)^{T} A^{T}\left(\lambda_{y}-\lambda_{x}\right)=0 .
$$

By applying $x$ and $y$ into (5), we have

$$
\begin{aligned}
& A y=b, \\
& A x=b .
\end{aligned}
$$

From these equations, we deduce that $A(y-x)=0$. So, we obtain

$$
(y-x)^{T} Q(y-x)=0 .
$$

2. Using the second-order Taylor expansion and the Hessian of the Lagrangian function of (P1), we get $q(y)=L\left(y, \lambda_{y}\right)=L\left(x, \lambda_{x}\right)+\nabla_{x} L\left(\bar{x}, \lambda_{x}\right)^{T}(y-x)+\frac{1}{2}(y-x)^{T} Q(y-x)$.

Since $(y-x)^{T} Q(y-x)=0 \quad \forall\left\{\left(x, \lambda_{x}\right),\left(y, \lambda_{y}\right)\right\} \subset \mathcal{K}_{\mathcal{F}}$. So, it follows that $q(x)=q(y)$.

We are able now to prove the following theorem of alternatives for quadratic programming with linear equality constraints.

Theorem 1. Suppose that the objective function $q$ is not constant on the feasible set $\mathcal{F}$, then for the problem (P1), only one of the following conditions is satisfied.

1) The objective function $q$ has no lower and no upper bound on the feasible set $\mathcal{F}$.

2) Every KKT point is a global minimizer.

3) Every KKT point is a global maximizer.

\section{Proof.}

Let $\alpha=\inf \left\{\frac{1}{2} x^{T} Q x-c^{T} x: A x=b\right\}$ and $\beta=\sup \left\{\frac{1}{2} x^{T} Q x-c^{T} x: A x=b\right\}$.

Suppose that the item 1) does not hold. This means that $\alpha$ or $\beta$ is finite.

i) Suppose that $\alpha$ is finite, by Frank-Wolfe Theorem [4], the problem (P1) admits a global minimizer $\bar{x}$ which is a KKT point and $\bar{\lambda}$ is the Lagrange multiplier associated. For any other pair $\left(x^{*}, \lambda^{*}\right) \in \mathcal{K}_{\mathcal{F}}$, we obtain $q(\bar{x})=q\left(x^{*}\right)$, from Lemma 2 . 
ii) Suppose that $\beta$ is finite. In the same way, we prove that every KKT point is a global maximizer.

iii) If $\alpha$ and $\beta$ are finite. From Lemma 2 we have that $\alpha=\beta$ and $\mathrm{q}$ is constant. Thus this case is excluded.

Now, we extend the previous results for the problem (P2).

Theorem 2. Suppose that the set $\mathcal{K}_{\triangle}$ of KKT triplets is convex, then

1) $\left(\bar{x}-x^{*}\right)^{T} Q\left(\bar{x}-x^{*}\right)=0 \quad \forall\left\{(\bar{x}, \bar{\lambda}, \bar{\mu}),\left(x^{*}, \lambda^{*}, \mu^{*}\right)\right\} \subset \mathcal{K}_{\triangle}$.

2) the Lagrangian function $\mathcal{L}$ and the objective function $q$ are constant over $\mathcal{K}_{\triangle}$.

Proof. Let $(\bar{x}, \bar{\lambda}, \bar{\mu})$ and $\left(x^{*}, \lambda^{*}, \mu^{*}\right)$ in $\mathcal{K}_{\triangle}$,

1) From the convexity of the set $\mathcal{K}_{\triangle}$ of KKT triplets and the complementary condition, we get, for every $t \in[0,1]$

$$
\begin{aligned}
t(\bar{x}, \bar{\lambda}, \bar{\mu})+(1-t)\left(x^{*}, \lambda^{*}, \mu^{*}\right) & = \\
& =\left(t \bar{x}+(1-t) x^{*}, t \bar{\lambda}+(1-t) \lambda^{*}, t \bar{\mu}+(1-t) \mu^{*}\right) \\
& =(x, \lambda, \mu) \in \mathcal{K}_{\Delta} .
\end{aligned}
$$

Let $i \in\{1,2, \ldots, n\}$ and $t \in[0,1]$, we have

$$
0=\mu_{i} x_{i}=\left(t \bar{\mu}_{i}+(1-t) \mu_{i}^{*}\right)\left(t \bar{x}_{i}+(1-t) x_{i}^{*}\right)=t(1-t)\left(\bar{\mu}_{i} x_{i}^{*}+\mu_{i}^{*} \bar{x}_{i}\right) .
$$

We obtain

$$
\bar{\mu}_{i} x_{i}^{*}=\mu_{i}^{*} \bar{x}_{i}=0 \quad \forall i=1,2, \ldots, n .
$$

Since $x^{*}$ and $\bar{x}$ satisfies

$$
\begin{gathered}
Q \bar{x}+A^{T} \bar{\lambda}-\bar{\mu}=c, \\
Q x^{*}+A^{T} \lambda^{*}-\mu^{*}=c,
\end{gathered}
$$

by substituting (13) into (12) gives us one equation

$$
Q\left(\bar{x}-x^{*}\right)+A^{T}\left(\bar{\lambda}-\lambda^{*}\right)-\left(\bar{\mu}-\mu^{*}\right)=0 .
$$

which is equivalent to

$$
Q\left(\bar{x}-x^{*}\right)=-A^{T}\left(\bar{\lambda}-\lambda^{*}\right)+\bar{\mu}-\mu^{*} .
$$

Multiply $\left(\bar{x}-x^{*}\right)$ by this relation, we get

$$
\left(\bar{x}-x^{*}\right)^{T} Q\left(\bar{x}-x^{*}\right)=-\left(\bar{x}-x^{*}\right)^{T} A^{T}\left(\bar{\lambda}-\lambda^{*}\right)+\left(\bar{x}-x^{*}\right)^{T}\left(\bar{\mu}-\mu^{*}\right) .
$$

Since $\left(\bar{x}-x^{*}\right)^{T} A^{T}=\left(A\left(\bar{x}-x^{*}\right)\right)^{T}=0$, we have from this equation that

$$
\left(\bar{x}-x^{*}\right)^{T} Q\left(\bar{x}-x^{*}\right)=-\sum_{i=1}^{n}\left(\bar{x}_{i} \mu_{i}^{*}+x_{i}^{*} \bar{\mu}_{i}\right) .
$$

and, from equation (11), we obtain

$$
\left(\bar{x}-x^{*}\right)^{T} Q\left(\bar{x}-x^{*}\right)=0 .
$$


2) From second-order Taylor expansion of the Lagrangian function $\mathcal{L}$ with respect to $x$, we get

$$
\mathcal{L}\left(x^{*}, \bar{\lambda}, \bar{\mu}\right)=\mathcal{L}(\bar{x}, \bar{\lambda}, \bar{\mu})+\nabla_{x} \mathcal{L}(\bar{x}, \bar{\lambda}, \bar{\mu})^{T}\left(x^{*}-\bar{x}\right)+\frac{1}{2}\left(x^{*}-\bar{x}\right)^{T} Q\left(x^{*}-\bar{x}\right)
$$

From (CN1) and (15) we have

$$
\mathcal{L}\left(x^{*}, \bar{\lambda}, \bar{\mu}\right)=\mathcal{L}(\bar{x}, \bar{\lambda}, \bar{\mu})
$$

Since

$$
\mathcal{L}\left(x^{*}, \bar{\lambda}, \bar{\mu}\right)=\frac{1}{2} x^{* T} Q x^{*}-c^{T} x^{*}-\sum_{i=1}^{n} x_{i}^{*} \bar{\mu}_{i}
$$

and

$$
\mathcal{L}(\bar{x}, \bar{\lambda}, \bar{\mu})=\frac{1}{2} \bar{x}^{T} Q \bar{x}-c^{T} \bar{x}
$$

Using equations (11) and (16), we get

$$
\frac{1}{2} x^{* T} Q x^{*}-c^{T} x^{*}=\frac{1}{2} \bar{x}^{T} Q \bar{x}-c^{T} \bar{x},
$$

and $q\left(x^{*}\right)=q(\bar{x})$.

Corollary 1. If a subset $\mathcal{K}$ of $\mathcal{K}_{\triangle}$ is convex, then the Lagrangian function $\mathcal{L}$ is constant over $\mathcal{K}$.

Next, we give a criteria for the convexity of the set of KKT triplets $\mathcal{K}_{\triangle}$. We begin with a first result concerning the sufficient conditions for the convexity of $\mathcal{K}_{\triangle}$.

Proposition 1. Suppose that one of the following conditions holds:

1) $d^{T} Q d \geq 0 \quad \forall d \in \operatorname{Ker}(\mathrm{A})$.

2) $I(\bar{x})=I\left(x^{*}\right) \quad \forall(\bar{x}, \bar{\lambda}, \bar{\mu}),\left(x^{*}, \lambda^{*}, \mu^{*}\right) \in \mathcal{K}_{\triangle}$.

then the set $\mathcal{K}_{\triangle}$ of KKT triplets is convex.

Proof. 1) Suppose that 1) holds and let $(\bar{x}, \bar{\lambda}, \bar{\mu})$ and $\left(x^{*}, \lambda^{*}, \mu^{*}\right)$ in $\mathcal{K}_{\triangle}$. We shall prove that, for every $t \in[0,1], t(\bar{x}, \bar{\lambda}, \bar{\mu})+(1-t)\left(x^{*}, \lambda^{*}, \mu^{*}\right) \in \mathcal{K}_{\triangle}$. It is sufficient to prove that the complementary condition holds. Since $\bar{x}-x^{*} \in \operatorname{Ker}(\mathrm{A})$,

$$
\left(\bar{x}-x^{*}\right)^{T} Q\left(\bar{x}-x^{*}\right) \geq 0
$$

and from equation (14), we have

$$
\left(\bar{x}-x^{*}\right)^{T} Q\left(\bar{x}-x^{*}\right)=-\sum_{i=1}^{n}\left(\bar{x}_{i} \mu_{i}^{*}+x_{i}^{*} \bar{\mu}_{i}\right) \leq 0 .
$$

This means that

$$
\left(\bar{x}-x^{*}\right)^{T} Q\left(\bar{x}-x^{*}\right)=0 .
$$

So $\bar{x}_{i} \mu_{i}^{*}+x_{i}^{*} \bar{\mu}_{i}=0 \quad \forall i=1,2, \ldots, n$.

From this condition, we have

$$
\left(t \bar{\mu}_{i}+(1-t) \mu_{i}^{*}\right)\left(t \bar{x}_{i}+(1-t) x_{i}^{*}\right)=t(1-t)\left(\bar{\mu}_{i} x_{i}^{*}+\mu_{i}^{*} \bar{x}_{i}\right)=0 \quad \forall i, \forall t \in[0,1] .
$$

We deduce that

$$
t(\bar{x}, \bar{\lambda}, \bar{\mu})+(1-t)\left(x^{*}, \lambda^{*}, \mu^{*}\right) \in \mathcal{K}_{\triangle} \quad \forall t \in[0,1] .
$$


2) Suppose that 2) holds and let $(\bar{x}, \bar{\lambda}, \bar{\mu})$ and $\left(x^{*}, \lambda^{*}, \mu^{*}\right)$ in $\mathcal{K}_{\triangle}$. We shall prove that, for every $t \in[0,1]$, then $t(\bar{x}, \bar{\lambda}, \bar{\mu})+(1-t)\left(x^{*}, \lambda^{*}, \mu^{*}\right) \in \mathcal{K}_{\triangle}$.

Since $I(\bar{x})=I\left(x^{*}\right)$ then, we have

$$
\bar{x}_{i} \mu_{i}^{*}=x_{i}^{*} \bar{\mu}_{i}=0 \quad \forall i=1,2, \ldots, n .
$$

This means that

$$
\bar{x}_{i} \mu_{i}^{*}+x_{i}^{*} \bar{\mu}_{i}=0 \quad \forall i=1,2, \ldots, n .
$$

So we have the set $\mathcal{K}_{\triangle}$ is convex.

From Proposition (1), we deduce the following corollary.

\section{Corollary 2.}

1) If $Q$ is positive semidefinite, then the set $\mathcal{K}_{\triangle}$ of KKT triplets is convex.

2) For every $J \subset\{1,2, \ldots, n\}, \mathcal{K}_{\triangle_{J}}=\left\{(x, \lambda, \mu) \in \mathcal{K}_{\triangle} \mid x \in \triangle_{J}\right\}$ is convex.

Theorem 3. Consider the following quadratic optimization problem

$$
\min \left\{\frac{1}{2} x^{T} Q x-c^{T} x: x \in \triangle_{J}\right\},
$$

where $Q$ is an arbitrary symmetric $n \times n$ matrix. Then, every local minimizer of problem (P3 is a global minimizer.

Proof. Let $x^{*}$ be a local minimizer for problem (P3 with Lagrange multiplier $\left(\lambda^{*}, \mu^{*}\right)$ and $x \in \triangle_{J}$. Then, we have $I(x)=I\left(x^{*}\right)$. This means that $\mu_{i}^{*} x_{i}=0 \quad \forall i=1,2, \ldots, n$ and $\mathcal{L}\left(x, \lambda^{*}, \mu^{*}\right)=q(x)$.

Note that $d=x-x^{*}$ which satisfy the following conditions

$$
d \in \operatorname{Ker}(\mathrm{A}) \text { and } d_{i}=0 \quad \forall i \in I\left(x^{*}\right)=J .
$$

Then, we deduce that

$$
d \in T_{\triangle_{J}}\left(x^{*}\right)=\left\{d \in \mathbb{R}^{n} \mid A d=0 ; d_{i}=0, \forall i \in I\left(x^{*}\right)=J\right\},
$$

this means that $d^{T} Q d \geq 0$. From second-order Taylor expansion of the Lagrangian function $\mathcal{L}$ with respect to $x$, we have

$$
q(x)=\mathcal{L}\left(x, \lambda^{*}, \mu^{*}\right)=\mathcal{L}\left(x^{*}, \lambda^{*}, \mu^{*}\right)+\nabla_{x} \mathcal{L}\left(x^{*}, \lambda^{*}, \mu^{*}\right)^{T} d+\frac{1}{2} d^{T} Q d .
$$

Hence, we obtain $q(x) \geq q\left(x^{*}\right)$. This completes the proof.

\section{Example}

By using our approach, we show that every local minimizer is a global minimizer for the following nonconvex quadratic minimization problems with linear constraints.

$$
\begin{array}{ll}
\min _{x \in \mathbb{R}^{5}} & q(x)=\frac{1}{2} x^{T} Q x, \\
\text { s. t. } & A x=\sum_{i=1}^{5} x_{i}=1, \\
& x_{i} \geq 0, \forall i \in\{1,2, \ldots, 5\},
\end{array}
$$


where $A=\left[\begin{array}{lllll}1 & 1 & 1 & 1 & 1\end{array}\right]$ and

$$
Q=\left[\begin{array}{rrrrr}
2 & 1 & 1 & 1 & -1 \\
1 & 2 & 1 & 1 & 1 \\
1 & 1 & 2 & 1 & -1 \\
1 & 1 & 1 & 2 & -1 \\
-1 & 1 & -1 & -1 & 2
\end{array}\right]
$$

The eigenvalues of $Q$ are: $\lambda_{1}=\frac{1}{2}(5-\sqrt{33})<0, \lambda_{2}=1>0, \lambda_{3}=1>0, \lambda_{4}=3>0$, $\lambda_{5}=\frac{1}{2}(5+\sqrt{33})>0$. Then this matrix is not positive semidefinite.

Let

$$
\Delta=\left\{x \in \mathbb{R}^{5} \mid \sum_{i=1}^{5} x_{i}=1 ; x_{i} \geq 0, i \in\{1,2, \ldots, 5\}\right\}
$$

and

$$
\begin{aligned}
\operatorname{Ker}(\mathrm{A}) & =\left\{d \in \mathbb{R}^{5} \mid \sum_{i=1}^{5} d_{i}=0\right\} \\
& =\left\{Z u \mid u \in \mathbb{R}^{4}\right\},
\end{aligned}
$$

where

$$
Z=\left[\begin{array}{rrrr}
1 & 0 & 0 & 0 \\
0 & 1 & 0 & 0 \\
0 & 0 & 1 & 0 \\
0 & 0 & 0 & 1 \\
-1 & -1 & -1 & -1
\end{array}\right]
$$

- First, we discuss according to the zero components of a solution of problem $(\mathrm{P})$. Then we can solve the necessary first order conditions (CN1) of this problem .

We obtain $\bar{x}=\left[\frac{3}{16}, 0, \frac{3}{16}, \frac{3}{16}, \frac{7}{16}\right]^{T} ; \quad \bar{\lambda}=\frac{5}{16} ; \quad \bar{\mu}=\left[0, \frac{11}{16}, 0,0,0\right]^{T}$.

- The solution $\bar{x}$ satisfies the conditions $(\mathrm{CN} 2)$ for problem $(\mathrm{P})$.

- Secondly, we show that, the matrix $Q$ is positive semidefinite over the set $\operatorname{Ker}(\mathrm{A})$. Indeed, we have

$$
y^{T} Q y \geq 0, \forall y \in \operatorname{Ker}(\mathrm{A}) \quad \Leftrightarrow \quad d^{T}\left(Z^{T} Q Z\right) d \geq 0, \forall d \in \mathbb{R}^{4} .
$$

The matrix $Z^{T} Q Z$ is positive semidefinite on $\mathbb{R}^{4}$, where

$$
Z^{T} Q Z=\left[\begin{array}{llll}
6 & 3 & 5 & 5 \\
3 & 2 & 3 & 3 \\
5 & 3 & 6 & 5 \\
5 & 3 & 5 & 6
\end{array}\right]
$$

and the eigenvalues of $Z^{T} Q Z$ are: $\lambda_{1}=9+2 \sqrt{19}>0, \lambda_{2}=1>0, \lambda_{3}=1>0$, $\lambda_{4}=9-2 \sqrt{19}>0$.

Therefore, the first item of Proposition 1 holds (i.e. the set $\mathcal{K}_{\triangle}$ of this problem is convex).

- From the second item of Theorem 2, then $\bar{x}$ is a global minimizer for $(\mathrm{P})$ 


\section{Conclusion}

We have given a new sufficient global optimality condition for non-convex quadratic minimization problems subjective to both linear equality and linear inequality constraints, related to the set of Karush-Kuhn-Tucker triplets (KKT) of this problem. We have shown that, if the set of KKT of this problem is convex, then a local minimum is global. Our approach can be a tool to solve globally a subclass of minimization of non-convex standard quadratic problems.

\section{Acknowledgement}

The authors would like to thank the referees for their unusually full and deep refereeing work, as well as valuable comments and helpful suggestions.

\section{References}

[1] Borwein, J. M. (1982). Necessary and sufficient conditions for quadratic minimality. Numerical Functional Analysis and Optimization, 5(2), 127-140. doi: 10.1080/01630568208816135

[2] Boyd, S. and Vandenberghe, L. (2004). Convex optimization. Cambridge University Press. doi: $10.1017 /$ cbo9780511804441

[3] Contesse, L. B. (1980). Une caracterisation complète des minima locaux en programmation quadratique. Numerische Mathematik, 34(3), 315-332. doi: 10.1007/BF01396705

[4] Frank, M. and Wolfe, P. (1956). An algorithm for quadratic programming. Naval Research Logistics Quarterly, 3(1-2), 95-110. doi: 10.1002/nav.3800030109

[5] Gould, N. I. M. Hribar, M. E. and Nocedal, J. (2001). On the solution of equality constrained quadratic programming arising in optimization. SIAM Journal on Scientific Computing, 23(4), 1376-1395. doi: 10.1137/S1064827598345667

[6] Lee, G. M. Tam, N. N. and Yen, N. D. (2005). Quadratic programming and affine variational inequalities: A qualitative study. Boston: Springer. doi: 10.1007/b105061

[7] Murty, K. G. and Kabadi, S. N. (1987). Some NP-complete problems in quadratic and nonlinear programming. Mathematical Programming, 39(2), 117-129. doi: 10.1007/BF02592948

[8] Nocedal, J. and Wright, S. J. (1999). Numerical optimization. New York: Springer. doi: 10.1007/978-0-387-40065-5

[9] Pardalos, P. M. and Rosen, J. B. (1987). Constrained global optimization: Algorithms and applications. Berlin: Springer. doi: 10.1007/BFb0000035

[10] Pardalos, P. M. and Rosen, J. B. (1988). Checking local optimality in quadratic programming is NP-hard. Operations Research Letters, 7(1), 33-35. doi: 10.1016/0167-6377(88)90049-1

[11] Strang, G. (1986). Introduction to applied mathematics. Cambridge Unniversity Press.

[12] Strang, G. (1988). A framework for equilibrium equations. SIAM Review, 30(2), 283-297. doi: $10.1137 / 1030048$ 